\title{
Editorial
}

\section{Who gives good weight-loss advice to the obese?}

According to a recent survey of 1873 obese (BMI at or above $30 \mathrm{~kg} / \mathrm{m}^{2}$ ) US adults, insufficient advice regarding weight loss is provided by health professionals ${ }^{(1)}$. Among those visiting a physician during the year before the survey, only 39\% were advised to lose weight. This number is actually slightly lower than in a previous study. Those with a poorer health and with higher BMI were more frequently advised to lose weight. The advice given was to lose more than $20 \%$ of body weight.

Clinical guidelines from the US Department of Health and Human Services (DHHS) ${ }^{(2)}$ clearly recommend both cutting energy intake and increasing physical activity level. Of the obese adults given advice in this study ${ }^{(1)}$, more than $40 \%$ were given advice not consistent with the US DHHS clinical guidelines, i.e. they were told either to cut intake or to increase their physical activity level. Clearly, as the authors conclude: 'Health professionals in this study do not take advantage of their influential role in promoting healthy behaviors among their obese patient population'.

\section{The problem}

This study points to a problem that health care all over the world seems to be facing: nobody gives advice since previous studies show that weight loss in already obese adults is close to a lost cause. Furthermore, several television shows in Europe as well as the USA are currently promoting rapid solutions such as gastric by-pass and subsequent plastic surgery. These are drastic measures, for which we do not have very much long-term evidence regarding health outcomes if we consider nutritional status as a whole. Some weight-loss drugs have been withdrawn in the past couple of years due to serious side-effects. Pharmaceutical companies are trying to find alternatives - which of course will be tremendously profitable for those who win the race.

We definitely need to get down to business and design joint strategies, including clinical guidelines as well as the use of these guidelines and an integration with public health policy, in order to support a healthy way out of the mess for these individuals. The option of leaving them to trust any rapid surgical solution (where they also will need longterm medical follow-up) or any weight-loss-diet-superherotrying-to-make-money who comes their way is a cop-out.

\section{The US guidelines}

In the USA, at least there are good guidelines ${ }^{(2)}$ on how to treat these patients. Yet these are apparently not followed.
Why is that? Looking closer at the guidelines reveals that the practical advice is to aim for a weight loss of $10 \%$ within 6 months and keep up the monitoring of the patient, and that guidance should be given regarding diet as well as physical activity. The guidelines provide a very comprehensive background for any action given, and also highlight a number of factors of importance in any treatment protocol: stimulus control, stress management, cognitive restructuring, social support, self-monitoring tools for eating/physical activity patterns and use of incentives. Maintenance of lost weight is of course a pivotal part of any successful weight loss and is discussed in detail in the document.

\section{The European and Australian perspective}

The European Charter on Counteracting Obesity ${ }^{(3)}$ is mostly looking at upstream activities such as policy interventions on the availability to healthy options, but in one paragraph states: 'Attention should also continue to be focused on preventing obesity in people who are already overweight and thus at high risk, and on treating the disease of obesity. Specific actions in this area would include: introducing timely identification and management of overweight and obesity in primary care, provision of training for health professionals in the prevention of obesity; and issuing clinical guidance for screening and treatment'.

The recent publication The Challenge of Obesity in the WHO European Region and the Strategies for Response $e^{(4)}$ states the following: "Current medical treatments for overweight and obesity in adults may be effective in the short term, thereby reducing associated risks. Surgical treatment, however, is the only intervention proven to maintain weight reduction in severely obese patients for the longer term (over 10 years). This weight reduction is associated with significant metabolic benefits: particularly a reduction in the incidence of type 2 diabetes. The treatment of overweight and obesity, for which appropriately trained health professionals are needed, requires not only weight reduction but also management of associated medical complications'.

The Obesity Management Taskforce for the European Association for Studies on Obesity published their European clinical guidelines last year ${ }^{(5)}$. These guidelines are consistent with the US guidelines and surgical treatment is mentioned down the line when other options 
have been tried. This document also adds the following comment regarding unorthodox approaches: 'Obesity treatment is often unsuccessful. As a result, unorthodox and unproven treatments flourish and are often offered. There is insufficient evidence to recommend in favor of herbal medicines, dietary supplements or homoeopathy for obesity management in the obese person. Physicians should advise patients to follow evidence-based treatments and recommend treatments only where evidence of safety and efficacy has been established'.

Australian guidelines were published in $2004^{(6)}$ and their approach is consistent with the American and European ones.

\section{Nutrition training for health-care staff}

At the risk of repeating myself, I ask: who is taking the responsibility of teaching health-care staff how to give dietary advice and who will assess the quality of the advice given? Training for health-care staff is suggested in the European Charter. But still, nothing has happened to address the lack of nutrition in the curricula for physicians as well as nurses, at least in Scandinavia ${ }^{(7)}$, and health-care staff in the USA seem to be having similar problems ${ }^{(1)}$. Citing Mowe et $a l .{ }^{(7)}$ : "The self-reported nutritional knowledge was inadequate among Scandinavian doctors and nurses. A combination of an integrated nutrition curriculum during the education, together with postgraduate training for both physicians and nurses should be established'.

\section{Clinical guidelines $v$. prevention policy documents}

There is a need for a more developed common strategy for making sure that the most important aspects of public health nutrition are taken into account, integrating the societal and public health aspects with the clinical work, and not treating clinical nutrition/obesity treatment as an entity on its own. Commonsense knowledge on adequate dietary advice and physical activity is not clinical.
Furthermore, why is the WHO European report highlighting surgical treatment more than anything else? Looking at the more general policy document from WHO Europe $\mathrm{e}^{(3,4)}$ in comparison with the clinical guidelines $^{(2,5,6)}$, it seems like no effort has been made to integrate these and different conclusions are being given. Could we perhaps be slightly more coherent in our approaches?

Agneta Yngve

Editor-in-Chief

\section{References}

1. Ko JY, Brown DR, Galuska DA, Zhang J, Blanck HM \& Ainsworth BE (2008) Weight loss advice US obese adults receive from health care professionals. Prev Med $\mathbf{4 7}$, 587-592.

2. National Institutes of Health (2008) Clinical Guidelines on the Identification, Evaluation, and Treatment of Overweight and Obesity in Adults: The Evidence Report. NIH Publication no. 98-4083. http://www.nhlbi.nih.gov/guide lines/obesity/ob_gdlns.pdf (accessed February 2009).

3. World Health Organization Regional Office for Europe (2006) The European Charter on Counteracting Obesity. http://www.euro.who.int/Document/E89567.pdf (accessed February 2009).

4. World Health Organization Regional Office for Europe (2007) The Challenge of Obesity in WHO European Region and the Strategies for Response. http://www.euro.who.int/ document/E90711.pdf (accessed February 2009).

5. Tsigosa C, Hainerb V, Basdevantc A et al., for the Obesity Management Taskforce of the European Association for the Study of Obesity (2008) Management of obesity in adults: European clinical practice guidelines. Obes Facts 1, $106-116$.

6. National Health and Medical Research Council, Australia (2003) Obesity Guidelines. Clinical Practice Guidelines for the Management of Overweight and Obesity in Adults. http://www.health.gov.au/internet/main/Publishing.nsf/ Content/obesityguidelines-guidelines-adults.htm/\$FILE/adults. pdf (accessed February 2009).

7. Mowe M, Bosaeus I, Rasmussen HH, Kondrup J, Unossson M, Rothenberg E \& Irtun $\varnothing$; Scandinavian Nutrition Group (2008) Insufficient nutritional knowledge among health care workers? Clin Nutr 27, 196-202. 